International Electronic Journal of Geometry

Volume 7 No. 1 PP. 108-125 (2014) (CIEJG

\title{
THEORY OF FINSLER SUBMANIFOLDS VIA BERWALD CONNECTION
}

\author{
AUREL BEJANCU AND HANI REDA FARRAN
}

Dedicated to memory of Proffessor Franki Dillen

\begin{abstract}
Let $\mathbb{F}^{m}=(M, F)$ be a Finsler submanifold of a Finsler manifold $\widetilde{\mathbb{F}}^{m+p}=(\widetilde{M}, \widetilde{F})$. By using the normal curvature vector field of $\mathbb{F}^{m}$ and the Berwald connections on both $\mathbb{F}^{m}$ and $\widetilde{\mathbb{F}}^{m+p}$, we obtain the structure equations for the immersion of $\mathbb{F}^{m}$ into $\widetilde{\mathbb{F}}^{m+p}$. This enables us to relate, for the first time in literature, the flag curvatures of $\mathbb{F}^{m}$ and $\widetilde{\mathbb{F}}^{m+p}$. Finally, we investigate the existence of totally geodesic Finsler submanifolds of a Randers $(c, K)$-sphere.
\end{abstract}

\section{INTRODUCTION}

The theory of Finsler submanifolds is as old as Finsler geometry is itself. Indeed, the Finsler geometry has emerged in 1918 when Finsler wrote his thesis on curves and surfaces in what he called generalized metric spaces. However, so far there is no well established theory of Finsler submanifolds as we have the theory of Riemannian submanifolds. Many reasons have concurred to this state of affairs in Finsler geometry. First, we mention that the geometry of Finsler manifolds is based on four classical Finsler connections: Berwald connection, Cartan connection, Chern-Rund connection and Hashiguchi connection. So, the question is: which one of these connections is more suitable for the theory of Finsler submanifolds? Secondly, most of the theories of Finsler submanifolds have been developed by using an induced nonlinear connection, which in general does not coincide with the canonical nonlinear connection of the submanifold. Finally, the structure equations obtained so far have cumbersome forms which are almost impossible to be used in a study of the geometry of a concrete Finsler submanifold. Several people have made some fundamental contributions to the subject: Akbar Zadeh [1], Barthel [3], Bejancu [4], [5], Bejancu-Farran [6], [7], Comic [8], Haimovici [9], Matsumoto [10], [11], Miron [13], Rund [14], Shen [15], Varga [17], Wegener [18].

The purpose of this paper is to develop a theory of Finsler submanifolds which does not make use of the induced nonlinear connection and the induced Finsler connection on a Finsler submanifold $\mathbb{F}^{m}=(M, F)$ of $\widetilde{\mathbb{F}}^{m+p}=(\widetilde{M}, \widetilde{F})$. To this end, we introduce the normal curvature vector field $\mathbf{n}$ of $\mathbb{F}^{m}$, and show that all structure

2010 Mathematics Subject Classification. Primary 53C60; Secondary 53C40.

Key words and phrases. Berwald connection, Finsler submanifold, flag curvature, normal curvature vector, Randers spheres, structure equations, totally geodesic Finsler submanifolds. 
equations of $\mathbb{F}^{m}$ are simply expressed in terms of some covariant derivatives of $\mathbf{n}$ (cf. (3.7), (3.16) and (3.17)). This leads us to a simple relation between the flag curvature of $\mathbb{F}^{m}$ and $\widetilde{\mathbb{F}}^{m+p}$ (cf. (3.19)). The main tool in our study is the Berwald connection on both $\mathbb{F}^{m}$ and $\widetilde{\mathbb{F}}^{m+p}$.

Now, we outline the content of the paper. In the first section we arrange some basic formulae from Finsler geometry and recall the main properties of vectorial Finsler connections (cf. Bejancu [5]). Next, in Section 2 we relate the Berwald connections on both $\mathbb{F}^{m}$ and $\widetilde{F}^{m+p}$ (cf. (2.23)). Then we introduce the normal curvature vector field $\mathbf{n}$ and show that all induced geometric objects on $\mathbb{F}^{m}$ are expressed in terms of the relative vertical covariant derivatives of $\mathbf{n}$ (cf. Theorem 2.1). In the last section we obtain the structure equations for a Finsler immersion, relating the curvature tensor fields of Berwald connections on $\mathbb{F}^{m}$ and $\widetilde{\mathbb{F}}^{m+p}$ (cf. Theorem 3.2). Also, we relate the flag curvatures of $\mathbb{F}^{m}$ and $\widetilde{\mathbb{F}}^{m+p}$ (cf. (3.19), (3.25)), and prove that a totally geodesic Finsler submanifold has the same flag curvature as its ambient Finsler manifold. Finally, we investigate the existence of totally geodesic submanifolds of Randers spheres.

The simple forms of the structure equations for a Finsler immersion, which are very rarely in Finsler geometry, lead us to the conclusion that the Berwald connection is the best for a study of the geometry of Finsler submanifolds.

\section{Preliminaries}

Let $M$ be an $m$-dimensional manifold and $T M$ be its tangent bundle. Then we take $\left(u^{\alpha}, v^{\alpha}\right)$ as local coordinates on $T M$, where $\left(u^{\alpha}\right)$ are the local coordinates on $M$ and $\left(v^{\alpha}\right)$ are the fiber coordinates. Suppose that there exists a function $F: T M \rightarrow[0, \infty)$, that is smooth on $T M^{\circ}=T M \backslash\{0\}$ and satisfies the conditions:

(i) $F$ is positively homogeneous of degree one with respect to $\left(v^{\alpha}\right)$, that is,

$$
F(u, k v)=k F(u, v), \text { for any } u \in M, v \in T_{u} M \text {, and } k>0 .
$$

(ii) The $m \times m$ matrix $\left[g_{\alpha \beta}(u, v)\right]$, where we put

$$
g_{\alpha \beta}(u, v)=\frac{1}{2} \frac{\partial^{2} F^{2}}{\partial v^{\alpha} \partial v^{\beta}},
$$

is a positive definite quadratic form for all $(u, v) \in T M^{\circ}$. Then $\mathbb{F}^{m}=(M, F)$ is called an $m$-dimensional Finsler manifold. Denote by $g^{\alpha \beta}$ the entries of the inverse matrix of $\left[g_{\alpha \beta}\right]$. The function $F$ and the Finsler tensor field $g=\left(g_{\alpha \beta}\right)$ are called the fundamental function and the Finsler metric of $\mathbb{F}^{m}$, respectively. In particular, if $\mathbb{F}^{m}$ is a Riemannian manifold, then $g$ becomes the Riemannian metric on $M$.

As all the geometric objects we work with are supposed to be smooth (differentiable of class $C^{\infty}$ ), we must consider them defined on $T M^{\circ}$. However, to simplify the notations, from now on we will omit the superscript "o" from $T M^{\circ}$. Denote by $\mathcal{F}(T M)$ the algebra of smooth functions on $T M$ and by $\Gamma(E)$ the $\mathcal{F}(T M)$-module of the smooth sections of a vector bundle $E$ over $T M$. Also, we use the Einstein convention, that is, repeated indices with one upper index and one lower index denotes summation over their range. If not stated otherwise, we shall use the following ranges for indices: $a, \beta, \gamma, \ldots \in\{1, \ldots, m\}, a, b, c, \ldots \in\{m+1, \ldots, m+p\}$, $i, j, k, \ldots \in\{1, \ldots, m+p\}$.

Now, we consider the kernel $V T M$ of the differential of the projection mapping $\Pi: T M \rightarrow M$, which is known as the vertical bundle over $T M$. Locally, $\Gamma(V T M)$ 
is spanned by $\left\{\partial / \partial v^{1}, \ldots, \partial / \partial v^{m}\right\}$. Then we consider the local vector fields

$$
\frac{\delta}{\delta u^{\alpha}}=\frac{\partial}{\partial u^{\alpha}}-G_{\alpha}^{\beta} \frac{\partial}{\partial v^{\beta}}
$$

where we put

$$
\text { (a) } G_{\alpha}^{\beta}=\frac{\partial G^{\beta}}{\partial v^{\alpha}}, \quad \text { (b) } G^{\beta}=\frac{1}{4} g^{\beta \gamma}\left\{\frac{\partial^{2} F^{2}}{\partial v^{\gamma} \partial u^{\varepsilon}} v^{\varepsilon}-\frac{\partial F^{2}}{\partial u^{\gamma}}\right\} .
$$

It is proved that there exists on $T M$ a complementary distribution $H T M$ to $V T M$ in $T T M$, which is locally spanned by $\left\{\delta / \delta u^{1}, \ldots, \delta / \delta u^{m}\right\}$. We call $H T M$ the canonical horizontal distribution on TM. In literature, HTM is also known as the canonical nonlinear connection on $T M$. Now, we consider the decomposition

$$
T T M=H T M \oplus V T M,
$$

and for any $X \in \Gamma(T T M)$ we put

$$
X=h X+v X,
$$

where $h$ and $v$ are the projection morphisms of TTM on HTM and VTM, respectively. Also, this decomposition of $T T M$ enables us to define the following almost product structure on $T M$ :

$$
Q\left(\frac{\delta}{\delta u^{\alpha}}\right)=\frac{\partial}{\partial v^{\alpha}}, \quad \text { and } \quad Q\left(\frac{\partial}{\partial v^{\alpha}}\right)=\frac{\delta}{\delta u^{\alpha}} .
$$

We call $Q$ the Finsler almost product structure on $T M$.

Next, we consider a Finsler connection $F C=(H T M, \nabla)$, where $H T M$ is the canonical horizontal distribution and $\nabla$ is a linear connection on the vertical bundle $V T M$. Then, we put

$$
\nabla_{\frac{\delta}{\delta u^{\beta}}} \frac{\partial}{\partial v^{\alpha}}=F_{\alpha}^{\gamma} \beta \frac{\partial}{\partial v^{\gamma}}, \quad \text { and } \quad \nabla_{\frac{\partial}{\partial v^{\beta}}} \frac{\partial}{\partial v^{\alpha}}=C_{\alpha}^{\gamma} \beta \frac{\partial}{\partial v^{\gamma}},
$$

and deduce that, locally, a Finsler connection is determined by the triple $\left(G_{\alpha}^{\gamma}, F_{\alpha}{ }_{\beta}, C_{\alpha}{ }_{\beta}\right)$. In literature there are four classical Finsler connections: Berwald connection, Cartan connection, Chern-Rund connection, and Hashiguchi connection. In the present paper we use only the Berwald connection $B F C=(H T M, \nabla)=\left(G_{\alpha}^{\gamma}, G_{\alpha}{ }_{\beta}, 0\right)$, that is, we have

$$
\begin{array}{ll}
\text { (a) } \nabla_{\frac{\delta}{\delta u^{\beta}}} \frac{\partial}{\partial v^{\alpha}}=G_{\alpha}^{\gamma} \frac{\partial}{\partial v^{\gamma}}, & \text { (b) } \nabla_{\frac{\partial}{\partial v^{\beta}}} \frac{\partial}{\partial v^{\alpha}}=0,
\end{array}
$$

where we put

$$
G_{\alpha}^{\gamma}{ }_{\beta}=\frac{\partial G_{\beta}^{\gamma}}{\partial v^{\alpha}} .
$$

Denote by $R$ the curvature tensor field of $\nabla$ and put
(a) $R\left(\frac{\delta}{\delta u^{\gamma}}, \frac{\delta}{\delta u^{\beta}}\right) \frac{\partial}{\partial v^{\alpha}}=H_{\alpha}{ }^{\mu}{ }_{\beta \gamma} \frac{\partial}{\partial v^{\mu}}$,
(b) $R\left(\frac{\partial}{\partial v^{\gamma}}, \frac{\delta}{\delta u^{\beta}}\right) \frac{\partial}{\partial v^{\alpha}}=G_{\alpha}{ }^{\mu}{ }_{\beta \gamma} \frac{\partial}{\partial v^{\mu}}$,
(c) $R\left(\frac{\partial}{\partial v^{\gamma}}, \frac{\partial}{\partial v^{\beta}}\right) \frac{\partial}{\partial v^{\alpha}}=S_{\alpha}{ }^{\mu}{ }_{\beta \gamma} \frac{\partial}{\partial v^{\mu}}$. 
Then we recall that (cf. Bejancu-Farran [7], p. 40, Matsumoto [12], pp. 118, 119)
(a) $H_{\alpha}{ }^{\mu}{ }_{\beta \gamma}=\frac{\delta G_{\alpha}{ }^{\mu} \beta}{\delta u^{\gamma}}-\frac{\delta G_{\alpha}{ }^{\mu} \gamma}{\delta u^{\beta}}+G_{\alpha}{ }^{\varepsilon}{ }_{\beta} G_{\varepsilon}{ }^{\mu}{ }_{\gamma}-G_{\alpha}{ }^{\varepsilon}{ }_{\gamma} G_{\varepsilon}{ }^{\mu}{ }_{\beta}=\frac{\partial R^{\mu}{ }_{\beta \gamma}}{\partial v^{\alpha}}$,
(b) $G_{\alpha}{ }_{\beta \gamma}=\frac{\partial G_{\alpha}^{\mu}{ }_{\beta}}{\partial v^{\gamma}}, \quad$ (c) $S_{\alpha}^{\mu}{ }_{\beta \gamma}=0$,

where

$$
R_{\beta \gamma}^{\mu}=\frac{\delta G_{\beta}^{\mu}}{\delta u^{\gamma}}-\frac{\delta G_{\gamma}^{\mu}}{\delta u^{\beta}} .
$$

Also, we recall the following Lie bracket formulae:

$$
\text { (a) }\left[\frac{\delta}{\delta u^{\beta}}, \frac{\delta}{\delta u^{\gamma}}\right]=R_{\beta \gamma}^{\mu} \frac{\partial}{\partial v^{\mu}}, \quad \text { (b) }\left[\frac{\delta}{\delta u^{\beta}}, \frac{\partial}{\partial v^{\gamma}}\right]=G_{\beta}{ }_{\gamma} \frac{\partial}{\partial v^{\mu}} \text {. }
$$

Due to (1.10a) we call $R^{\mu}{ }_{\beta \gamma}$ the integrability tensor field of the canonical horizontal distribution HTM.

The Cartan and Chern-Rund connections have $F_{\alpha}^{\gamma}{ }_{\beta}$ given by

$$
F_{\alpha}^{\gamma}{ }^{\gamma}=\frac{1}{2} g^{\gamma \varepsilon}\left\{\frac{\delta g_{\varepsilon \alpha}}{\delta u^{\beta}}+\frac{\delta g_{\varepsilon \beta}}{\delta u^{\alpha}}-\frac{\delta g_{\alpha \beta}}{\delta u^{\varepsilon}}\right\} \text {. }
$$

Also, the Cartan tensor field $g_{\alpha \beta \gamma}$ given by

$$
g_{\alpha \beta \gamma}=\frac{1}{2} \frac{\partial g_{\alpha \beta}}{\partial v^{\gamma}}
$$

has a great role in Finsler geometry. It is important to note that the Berwald connection is neither $h$-metrical, nor $v$-metrical connection. More precisely, we have

$$
\text { (a) } g_{\alpha \beta \mid \gamma}=2 g_{\alpha \varepsilon}\left(F_{\beta}^{\varepsilon}{ }_{\gamma}-G_{\beta}^{\varepsilon}{ }_{\gamma}\right), \quad \text { (b) } g_{\alpha \beta \| \gamma}=2 g_{\alpha \beta \gamma},
$$

where "|" and "|" represent the horizontal and vertical covariant derivatives with respect to Berwald connection. The homogeneity of the fundamental function implies some useful identities:

$$
\begin{array}{lll}
\text { (a) } \quad F_{\beta}{ }^{\varepsilon}{ }_{\gamma} v^{\gamma}=G_{\beta}^{\varepsilon}, & \text { (b) } \quad G_{\beta}{ }^{\varepsilon}{ }_{\gamma} v^{\gamma}=G_{\beta}^{\varepsilon}, \quad \text { (c) } \quad G_{\beta}^{\varepsilon} v^{\beta}=2 G^{\varepsilon}, \\
\text { (d) } \quad g_{\alpha \beta \gamma} v^{\gamma}=0, \quad \text { (e) } \quad H_{\alpha}{ }^{\mu}{ }_{\beta \gamma} v^{\alpha}=R_{\beta \gamma}^{\mu}, \quad \text { (f) } \quad G_{\alpha}{ }^{\mu}{ }_{\beta \gamma} v^{\alpha}=0 .
\end{array}
$$

Then, by using (1.12a), (1.13a) and (1.13b), we deduce that

$$
g_{\alpha \beta \mid \gamma} v^{\gamma}=0
$$

Now, we want to introduce the flag curvature of $\mathbb{F}^{m}$. Let $(u, v)$ be a point of $T M$, where $u=\left(u^{\alpha}\right)$ is a point of $M$ and $v=\left(v^{\alpha}\right)$ is a non-zero tangent vector to $M$ at $u$. Suppose that $X=\left(X^{\alpha}\right)$ is another tangent vector to $M$ at $u$ such that $v$ and $X$ are linearly independent in $T_{u} M$. Then, according to Bao-Chern-Shen [2], p. 68 , we call the plane $\Pi(X)=\operatorname{span}\{v, X\}$ the flag at $u$ with flagpole $v$ and transverse edge $X$. Then the flag curvature of $\mathbb{F}^{m}$ at the point $u$ with respect to the flag $\Pi(X)$ is given by

$$
K(X)=\frac{H_{\alpha \varepsilon \beta \gamma} v^{\alpha} v^{\beta} X^{\varepsilon} X^{\gamma}}{F^{2} h_{\varepsilon \gamma} X^{\varepsilon} X^{\gamma}},
$$

where $H_{\alpha \varepsilon \beta \gamma}=g_{\mu \varepsilon} H_{\alpha}{ }^{\mu}{ }_{\beta \gamma}$, and $h_{\varepsilon \gamma}$ are the local components of the angular metric given by

$$
h_{\varepsilon \gamma}=g_{\varepsilon \gamma}-\ell_{\varepsilon} \ell_{\gamma}, \quad \ell_{\varepsilon}=g_{\varepsilon \mu} \frac{v^{\mu}}{F} .
$$


Next, we put
(a) $R_{\alpha \beta \gamma}=g_{\alpha \mu} R_{\beta \gamma}^{\mu}$,
(b) $R_{\alpha \beta \gamma} v^{\beta}=R_{\alpha \gamma}$,

and note that $R_{\alpha \gamma}$ is a symmetric Finsler tensor field. Then, by using (1.13e), (1.16) and (1.15) we deduce that

$$
K(X)=\frac{R_{\varepsilon \gamma} X^{\varepsilon} X^{\gamma}}{F^{2} h_{\varepsilon \gamma} X^{\varepsilon} X^{\gamma}} .
$$

Denoting the denominator in (1.17) by $\Delta(X)$, it is easy to see that we have

$$
\Delta(X)=g(X, X) g(v, v)-g(v, X)^{2} .
$$

\section{Induced Geometric Objects on a Finsler Submanifold}

Let $\widetilde{\mathbb{F}}^{m+p}=(\widetilde{M}, \widetilde{F})$ be an $(m+p)$-dimensional Finsler manifold and $M$ be an $m$-dimensional submanifold of $\widetilde{M}$. Take $\left(x^{i}, y^{i}\right)$ and $\left(u^{\alpha}, v^{\alpha}\right)$ as local coordinates on $T \widetilde{M}$ and $T M$ respectively, and suppose that the immersion of $M$ in $\widetilde{M}$ is locally given by the equations

$$
x^{i}=x^{i}\left(u^{1}, \ldots, u^{m}\right), \quad i \in\{1, \ldots, m+p\} .
$$

To simplify the equations involved in the study, we make the notations:

$$
B_{\alpha}^{i}=\frac{\partial x^{i}}{\partial u^{\alpha}}, \quad B_{\alpha \beta}^{i}=\frac{\partial^{2} x^{i}}{\partial u^{\alpha} \partial u^{\beta}}, \quad B_{\alpha \beta \gamma}^{i}=\frac{\partial^{3} x^{i}}{\partial u^{\alpha} \partial u^{\beta} \partial u^{\gamma}} .
$$

The fundamental function $\widetilde{F}$ of $\widetilde{\mathbb{F}}^{m+p}$ induces a function on $T M$ as follows:

$$
F\left(u^{\alpha}, v^{\alpha}\right)=\widetilde{F}\left(x^{i}(u), y^{i}(u, v)\right),
$$

where we set

$$
y^{i}(u, v)=B_{\alpha}^{i} v^{\alpha} .
$$

It is easy to check that $F$ defines a Finsler structure on $M$. Then we say that $\mathbb{F}^{m}=(M, F)$ is a Finsler submanifold of $\widetilde{\mathbb{F}}^{m+p}$. The Finsler metrics $g=\left(g_{\alpha \beta}(u, v)\right)$ and $\widetilde{g}=\left(\widetilde{g}_{i j}(x, y)\right)$ are related by

$$
g_{\alpha \beta}(u, v)=\widetilde{g}_{i j}(x(u), y(u, v)) B_{\alpha}^{i} B_{\beta}^{j} .
$$

Remark 2.1. The geometric objects defined for $\mathbb{F}^{m}$ in the previous section will be considered for $\widetilde{\mathbb{F}}^{m+p}$, but with a tilde, as for example: $G_{\alpha}^{\gamma}, G_{\alpha}{ }_{\beta}, R^{\gamma}{ }_{\alpha \beta}$ become $\widetilde{G}_{i}^{k}, \widetilde{G}_{i}{ }^{k}{ }_{j}, \widetilde{R}^{k}{ }_{i j}$, respectively

Next, by using (2.2) we deduce that the natural field of frames $\left\{\partial / \partial u^{\alpha}, \partial / \partial v^{\alpha}\right\}$ and $\left\{\partial / \partial x^{i}, \partial / \partial y^{i}\right\}$ on $T M$ and $T \widetilde{M}$, are related by

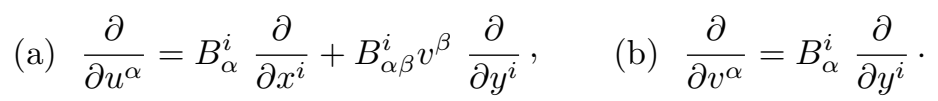

Then we consider the Finsler metrics $\widetilde{g}=\left(\widetilde{g}_{i j}\right)$ and $g=\left(g_{\alpha \beta}\right)$ of $\widetilde{F}^{m+p}$ and $\mathbb{F}^{m}$, as Riemannian metrics on the vertical bundles $V T \widetilde{M}$ and $V T M$, respectively. Due to (2.3) and (2.4b) we deduce that $g$ is the induced Riemannian metric on $V T M$ by $\widetilde{g}$. This enables us to consider the complementary orthogonal vector subbundle $V T M^{\perp}$ to $V T M$ in $V T \widetilde{M}$ restricted to $T M$. Thus we have the orthogonal decomposition

$$
V T \widetilde{M}_{\mid T M}=V T M \oplus V T M^{\perp} .
$$

We call $V T M^{\perp}$ the Finsler normal bundle for the immersion of $\mathbb{F}^{m}$ into $\widetilde{F}^{m+p}$. 
Now, we choose a local field of orthonormal frames with respect to $\widetilde{g}$ in $\Gamma\left(V T M^{\perp}\right)$ :

$$
N_{a}=N_{a}^{i} \frac{\partial}{\partial y^{i}}, \quad a \in\{m+1, \ldots, m+p\} .
$$

Thus we have
(a) $\widetilde{g}_{i j} B_{\alpha}^{i} N_{a}^{j}=0$,
(b) $\widetilde{g}_{i j} N_{a}^{i} N_{b}^{j}=\delta_{a b}$.

If $\left[\begin{array}{c}\widetilde{B}_{i}^{\alpha} \\ \widetilde{N}_{i}^{a}\end{array}\right]$ is the inverse of the matrix $\left[\begin{array}{ll}B_{\alpha}^{i} & N_{a}^{i}\end{array}\right]$, then we have:
(a) $\widetilde{B}_{i}^{\alpha} B_{\beta}^{i}=\delta_{\beta}^{\alpha}$,
(b) $\widetilde{B}_{i}^{\alpha} N_{a}^{i}=0$
(c) $\tilde{N}_{i}^{a} B_{\alpha}^{i}=0$
(d) $\tilde{N}_{i}^{a} N_{b}^{i}=\delta_{b}^{a}$,
(e) $B_{\alpha}^{i} \widetilde{B}_{j}^{\alpha}+N_{a}^{i} \widetilde{N}_{j}^{a}=\delta_{j}^{i}$.

By using (2.3), (2.6) and (2.7e) we deduce that
(a) $\widetilde{B}_{i}^{\alpha}=\widetilde{g}_{i j} B_{\beta}^{j} g^{\beta \alpha}$,
(b) $\tilde{N}_{j}^{a}=\widetilde{g}_{i j} N_{b}^{j} \delta^{b a}$,
(c) $g^{\alpha \beta}=\widetilde{g}^{i j} \widetilde{B}_{i}^{\alpha} \widetilde{B}_{j}^{\beta}$.

Also, the decomposition (2.5) is locally expressed as follows:

$$
\frac{\partial}{\partial y^{i}}=\widetilde{B}_{i}^{\alpha} \frac{\partial}{\partial v^{\alpha}}+\widetilde{N}_{i}^{a} N_{a}
$$

Next, we consider the Cartan tensor fields of both $\mathbb{F}^{m}$ and $\widetilde{F}^{m+p}$, and obtain
(a) $g_{\alpha \beta \gamma}=\widetilde{g}_{i j k} B_{\alpha}^{i} B_{\beta}^{j} B_{\gamma}^{k}$,
(b) $g_{\alpha}{ }^{\gamma}{ }_{\beta}=\widetilde{g}_{i}{ }^{k}{ }_{j} B_{\alpha}^{i} B_{\beta}^{j} \widetilde{B}_{k}^{\gamma}$.

Remark 2.2. Throughout the paper we shall use $g_{\alpha \beta}, g^{\alpha \beta}, \widetilde{g}_{i j}, \widetilde{g}^{i j}, \delta_{a b}$ and $\delta^{a b}$ for lowering or raising indices for mixed Finsler tensor fields with local components $T_{\beta j b \ldots}^{\alpha i a \ldots}$

We also need the following mixed Finsler tensor field

$$
g_{a \alpha \beta}=\widetilde{g}_{i j k} N_{a}^{i} B_{\alpha}^{j} B_{\beta}^{k}
$$

Contracting (2.11) by $\widetilde{N}_{h}^{a}$ and using $(2.7 \mathrm{e})$, we obtain

$$
g_{a \alpha \beta} \widetilde{N}_{h}^{a}=\widetilde{g}_{h j k} B_{\alpha}^{j} B_{\beta}^{k}-g_{\alpha \beta \gamma} \widetilde{B}_{h}^{\gamma} .
$$

Now, from (2.8a) we deduce that

$$
g_{\alpha \beta} \widetilde{B}_{i}^{\beta}=\widetilde{g}_{i j} B_{\alpha}^{j} .
$$

Taking partial derivatives of this equation with respect to $v^{\gamma}$ and using (1.11) for Cartan tensor fields of both $\mathbb{F}^{m}$ and $\widetilde{F}^{m+p}$, and (2.12), we infer that

$$
\frac{\partial \widetilde{B}_{i}^{\beta}}{\partial v^{\gamma}}=2 g_{a}^{\beta}{ }_{\gamma} \widetilde{N}_{i}^{a}
$$

Some basic identities on a Finsler manifold are stated in the next proposition.

Proposition 2.1. Let $\mathbb{F}^{m}$ be a Finsler submanifold of $\widetilde{\mathbb{F}}^{m+p}$. Then we have

$$
G_{\beta}^{\alpha} B_{\alpha}^{h}+H_{\beta}^{a} N_{a}^{h}=B_{\beta \varepsilon}^{h} v^{\varepsilon}+\widetilde{G}_{j}^{h} B_{\beta}^{j}+D_{\beta}^{\alpha} B_{\alpha}^{h},
$$

where we put
(a) $H_{\beta}^{a}=\widetilde{N}_{k}^{a}\left(B_{\beta \varepsilon}^{k} v^{\varepsilon}+\widetilde{G}_{j}^{k} B_{\beta}^{j}\right)$,
(b) $D_{\beta}^{\alpha}=g_{a}{ }^{\alpha}{ }_{\beta} H_{\varepsilon}^{a} v^{\varepsilon}$. 
Proof. By using (1.2b) for both $\mathbb{F}^{m}$ and $\widetilde{\mathbb{F}}^{m+p}$, and taking into account (2.1), (2.4), (2.8), (2.7e) and (2.6a), we obtain

$$
2 G^{\alpha}=\widetilde{B}_{k}^{\alpha}\left(2 \widetilde{G}^{k}+B_{\gamma \varepsilon}^{k} v^{\gamma} v^{\varepsilon}\right) .
$$

Then, taking the derivatives of this equation with respect to $v^{\beta}$ and using (1.2a) for both $\mathbb{F}^{m}$ and $\widetilde{\mathbb{F}}^{m+p},(2.13)$ and $(2.4 \mathrm{~b})$, we deduced that

$$
G_{\beta}^{\alpha}=g_{a}{ }_{\beta}{ }_{\beta} \widetilde{N}_{k}^{a}\left(2 \widetilde{G}^{k}+B_{\gamma \varepsilon}^{k} v^{\gamma} v^{\varepsilon}\right)+\widetilde{B}_{k}^{\alpha}\left(\widetilde{G}_{j}^{k} B_{\beta}^{j}+B_{\beta \varepsilon}^{k} v^{\varepsilon}\right) .
$$

On the other hand, taking into account that $\widetilde{G}^{k}$ are positively homogeneous functions of degree two with respect to $y^{i}$ (see (1.9c)), and using (2.2) we obtain

$$
2 \widetilde{G}^{k}=\frac{\partial \widetilde{G}^{k}}{\partial y^{j}} y^{j}=\widetilde{G}_{j}^{k} B_{\gamma}^{j} v^{\gamma} .
$$

Hence, by using (2.18) and (2.15) into (2.17), we infer that

$$
G_{\beta}^{\alpha}=D_{\beta}^{\alpha}+\widetilde{B}_{k}^{\alpha}\left(\widetilde{G}_{j}^{k} B_{\beta}^{j}+B_{\beta \varepsilon}^{k} v^{\varepsilon}\right) .
$$

Finally, contracting (2.19) by $B_{\alpha}^{h}$ and $(2.15 \mathrm{a})$ by $N_{a}^{h}$, and then adding the two equalities we obtain (2.14) via (2.7e).

Now, we are able to express $\left\{\delta / \delta u^{\alpha}\right\}$ given by (1.1) in terms of $\left\{\delta / \delta x^{i}, \partial / \partial y^{i}\right\}$. Indeed, by using (1.1) for both $\mathbb{F}^{m}$ and $\widetilde{\mathbb{F}}^{m+p},(2.4)$ and (2.14) we deduce that the canonical horizontal distribution $H T M$ of $\mathbb{F}^{m}$ is locally spanned by

$$
\frac{\delta}{\delta u^{\alpha}}=B_{\alpha}^{i} \frac{\delta}{\delta x^{i}}+\left(H_{\alpha}^{a} N_{a}^{i}-D_{\alpha}^{\gamma} B_{\gamma}^{i}\right) \frac{\partial}{\partial y^{i}} .
$$

Also, by using (2.7b), (2.7d) and (2.13), we deduce that

$$
\begin{aligned}
\widetilde{B}_{h}^{\alpha} \frac{\partial}{\partial v^{\gamma}}\left(H_{\beta}^{a} N_{a}^{h}\right) & =-\left(H_{\beta}^{a} N_{a}^{h}\right) \frac{\partial \widetilde{B}_{h}^{\alpha}}{\partial v^{\gamma}} \\
& =-2 H_{\beta}^{a} g_{b}{ }^{\alpha} \gamma\left(\widetilde{N}_{h}^{b} N_{a}^{h}\right)=-2 H_{\beta}^{a} g_{a}{ }^{\alpha}{ }_{\gamma} .
\end{aligned}
$$

Next, let $B F C=(H T M, \nabla)=\left(G_{\beta}^{\alpha}, G_{\beta}{ }^{\alpha}{ }_{\gamma}, 0\right)$ and $\widetilde{B F C}=(H T \widetilde{M}, \widetilde{\nabla})=$ $\left(\widetilde{G}_{j}^{i}, \widetilde{G}_{j}{ }^{i}{ }_{k}, 0\right)$ be the Berwald connections of $\mathbb{F}^{m}$ and $\widetilde{\mathbb{F}}^{m+p}$. Thus according to (1.5) we have

$$
\begin{array}{ll}
\text { (a) } \widetilde{\nabla}_{\frac{\delta}{\delta x^{k}}} \frac{\partial}{\partial y^{j}}=\widetilde{G}_{j}{ }^{i} \frac{\partial}{\partial y^{i}}, & \text { (b) } \widetilde{\nabla}_{\frac{\partial}{\partial y^{k}}} \frac{\partial}{\partial y^{j}}=0 .
\end{array}
$$

Then we prove the following.

Proposition 2.2. The local coefficients $G_{\beta}{ }^{\alpha}{ }_{\gamma}$ and $\widetilde{G}_{j}{ }^{i}{ }_{k}$ of the Berwald connections of $\mathbb{F}^{m}$ and $\widetilde{\mathbb{F}}^{m+p}$ are related by

$$
\left(G_{\beta}{ }^{\alpha}{ }_{\gamma}+D_{\beta}{ }^{\alpha}{ }_{\gamma}\right) B_{\alpha}^{i}+H_{\beta}{ }^{b}{ }_{\gamma} N_{b}^{i}=B_{\beta \gamma}^{i}+\widetilde{G}_{j}{ }^{i}{ }_{k} B_{\beta}^{j} B_{\gamma}^{k},
$$

where we put
(a) $D_{\beta}{ }^{\alpha}{ }_{\gamma}=-\frac{\partial D_{\beta}^{\alpha}}{\partial v^{\gamma}}-2 H_{\beta}^{a} g_{a}^{\alpha}{ }_{\gamma}$
(b) $H_{\beta}{ }^{b} \gamma=\widetilde{N}_{h}^{b} \frac{\partial}{\partial v^{\gamma}}\left(H_{\beta}^{a} N_{a}^{h}\right)$. 
Proof. First, we take the derivatives of (2.14) with respect to $v^{\gamma}$ and by using (1.6) for both $\mathbb{F}^{m}$ and $\widetilde{\mathbb{F}}^{m+p}$ and (2.4b), we obtain

$$
\left(G_{\beta}{ }^{\varepsilon} \gamma-\frac{\partial D_{\beta}^{\varepsilon}}{\partial v^{\gamma}}\right) B_{\varepsilon}^{h}+\frac{\partial}{\partial v^{\gamma}}\left(H_{\beta}^{a} N_{a}^{h}\right)=B_{\beta \gamma}^{h}+\widetilde{G}_{j}{ }^{h}{ }_{k} B_{\beta}^{j} B_{\gamma}^{k}
$$

Contracting this in turn by $\widetilde{B}_{h}^{\alpha}$ and $\widetilde{N}_{h}^{b}$, and using (2.7a), (2.21), (2.24), and (2.7c), we deduce that

$$
\begin{aligned}
& \text { (a) } G_{\beta}{ }^{\alpha}{ }_{\gamma}+D_{\beta}{ }^{\alpha}{ }_{\gamma}=\widetilde{B}_{h}^{\alpha}\left(B_{\beta \gamma}^{h}+\widetilde{G}_{j}{ }^{h}{ }_{k} B_{\beta}^{j} B_{\gamma}^{k}\right), \\
& \text { (b) } H_{\beta}{ }^{b}{ }_{\gamma}=\widetilde{N}_{h}^{b}\left(B_{\beta \gamma}^{h}+\widetilde{G}_{j}{ }^{h}{ }_{k} B_{\beta}^{j} B_{\gamma}^{k}\right) .
\end{aligned}
$$

Finally, contracting $(2.25 \mathrm{a})$ by $B_{\alpha}^{i}$ and $(2.25 \mathrm{~b})$ by $N_{b}^{i}$, and then adding, we obtain (2.23) via $(2.7 \mathrm{e})$.

Now, we define a tensor field $D$ on $\mathbb{F}^{m}$ by

$$
D\left(\frac{\partial}{\partial v^{\gamma}}, \frac{\partial}{\partial v^{\beta}}\right)=D_{\beta}^{\alpha}{ }_{\gamma} \frac{\partial}{\partial v^{\alpha}}
$$

and a mixed Finsler tensor field $H$ on $\mathbb{F}^{m}$ with respect to the Finsler normal bundle $V T M^{\perp}$, as follows

$$
H\left(\frac{\partial}{\partial v^{\gamma}}, \frac{\partial}{\partial v^{\beta}}\right)=H_{\beta}{ }^{a}{ }^{\prime} N_{a} .
$$

Then, by using (2.20), (2.4b), (2.22), (1.5), (2.26) and (2.27) we deduce that (2.23) is equivalent to

$$
\widetilde{\nabla}_{h X} v Y=\nabla_{h X} v Y+D(Q h X, v Y)+H(Q h X, v Y),
$$

for any $X, Y \in \Gamma(T T M)$, where $h$ and $v$ are defined by (1.3) and $Q$ is the Finsler almost product structure on $T M$ given by (1.4).

According to the terminology from the theory of Riemannian submanifolds, we call (2.28) (or, equivalently, (2.23)) the Gauss formulae for the Finsler immersion of $\mathbb{F}^{m}$ in $\widetilde{\mathbb{F}}^{m+p}$. Also, we note that the difference

$$
\widetilde{\nabla}_{h X} v Y-\nabla_{h X} v Y
$$

is determined by a Finsler tangent vector field $D(Q h X, v Y)$ (i.e., section of $V T M)$, and a Finsler normal vector field $H(Q h X, v Y)$ (i.e., section of $\left.V T M^{\perp}\right)$. For this reason we call $D$ given by $(2.26)$ and $(2.24 \mathrm{a})$, and $H$ given by $(2.27),(2.25 \mathrm{~b})$ and $(2.24 \mathrm{~b})$, the tangent second fundamental form and the normal second fundamental form, respectively. Moreover, we have the following.

\section{Proposition 2.3.}

(i) $D_{\beta}{ }^{\alpha}{ }_{\gamma}$ and $H_{\beta}{ }^{a}{ }_{\gamma}$ are symmetric Finsler tensor fields satisfying the identities

$$
\text { (a) } D_{\beta}{ }^{\alpha}{ }_{\gamma} v^{\gamma}=-D_{\beta}^{\alpha}, \quad \text { (b) } D_{\beta}{ }^{\alpha}{ }_{\gamma} v^{\beta} v^{\gamma}=-D_{\beta}^{\alpha} v^{\beta}=0 \text {, }
$$

(c) $H_{\beta}{ }^{a}{ }_{\gamma} v^{\gamma}=H_{\beta}^{a}$.

(ii) The Finsler tensor fields $D_{\beta}{ }^{\alpha} \gamma$ and $D_{\beta}^{\alpha}$ are positively homogeneous of degrees 0 and 1 respectively, with respect to $\left(v^{\varepsilon}\right)$. 
Proof. (i) From (2.25) we see that both $D_{\beta}{ }^{\alpha}{ }_{\gamma}$ and $H_{\beta}{ }^{a}{ }_{\gamma}$ are symmetric Finsler tensor fields with respect to $(\beta \gamma)$. Next, contracting $(2.25 \mathrm{a})$ by $v^{\gamma}$ and using $(1.13 \mathrm{~b})$ and (2.19) we obtain (2.29a). Similarly, contracting (2.19) by $v^{\beta}$ and using (1.13c), (2.16) and (2.29a) we deduce (2.29b). Finally, $(2.29 \mathrm{c})$ is a consequence of $(2.25 \mathrm{~b})$ and (2.15a). (ii) By using (2.13) we deduce that $\widetilde{B}_{i}^{\alpha}$ are positively homogeneous of degree 0 . Then, from $(2.25 \mathrm{a})$, it follows that $D_{\beta}{ }^{\alpha}{ }_{\gamma}$ are positively homogeneous of degree 0 . Finally, from $(2.29 \mathrm{a})$ we conclude that $D_{\beta}^{\alpha}$ are positively homogeneous of degree 1 .

Next, we make the notation

$$
H_{0}^{a}=H_{\beta}^{a} v^{\beta}=H_{\beta}{ }^{a}{ }_{\gamma} v^{\beta} v^{\gamma},
$$

and define on each coordinate neighbourhood of $T M$ the section $\mathbf{n}$ of $V T M^{\perp}$ by

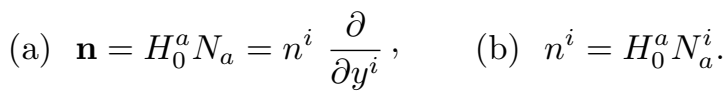

Then it is easy to check that $\mathbf{n}$ defines a global section of $V T M^{\perp}$, that is, the local formula (2.31a) is invariant with respect to both the change of local coordinates on $T M$ and the change of orthonormal basis in $\Gamma\left(V T M^{\perp}\right)$. As $H_{0}^{a} / F^{2}, a \in\{m+$ $1, \ldots, m+p\}$, are called the normal curvatures of $\mathbb{F}^{m}$ (cf. Bejancu [4]), we call $\mathbf{n}$ the normal curvature vector field of $\mathbb{F}^{m}$. As we shall see in the next theorem, the normal curvature vector field $\mathbf{n}$ determines all the induced geometric objects of the Finsler immersion of $\mathbb{F}^{m}$ into $\widetilde{\mathbb{F}}^{m+p}$.

The following notations will simplify the presentation:

$$
\text { (a) } n_{\alpha}^{i}=\frac{1}{2} \frac{\partial n^{i}}{\partial v^{\alpha}}, \quad \text { (b) } n_{\alpha \beta}^{i}=\frac{1}{2} \frac{\partial^{2} n^{i}}{\partial v^{\alpha} \partial v^{\beta}}
$$

$$
\text { (c) } n_{\alpha \beta \gamma}^{i}=\frac{1}{2} \frac{\partial^{3} n^{i}}{\partial v^{\alpha} \partial v^{\beta} \partial v^{\gamma}} \text {. }
$$

Note that $n_{\alpha}^{i}, n_{\alpha \beta}^{i}$ and $n_{\alpha \beta \gamma}^{i}$ are the local components of some mixed Finsler tensor fields on $\mathbb{F}^{m}$ with respect to the vector bundle $V T \widetilde{M}_{\mid T M}$ (cf. Bejancu [5], p. 34). Now, we can state the main result of this section.

Theorem 2.1. Let $\mathbb{F}^{m}$ be a Finsler submanifold of $\widetilde{\mathbb{F}}^{m+p}$. Then the induced geometric objects $H_{\beta}^{a}, D_{\beta}^{\alpha}, H_{\beta}{ }^{a}{ }_{\gamma}$, and $D_{\beta}{ }^{\alpha}{ }_{\gamma}$ are expressed in terms of $n_{\beta}^{i}$ and $n_{\beta \gamma}^{i}$ as follows:

$$
\begin{array}{ll}
\text { (a) } H_{\beta}^{a}=\widetilde{N}_{i}^{a} n_{\beta}^{i}, & \text { (b) } D_{\beta}^{\alpha}=\widetilde{B}_{i}^{\alpha} n_{\beta}^{i}, \\
\text { (c) } H_{\beta}{ }^{a}{ }_{\gamma}=\widetilde{N}_{i}^{a} n_{\beta \gamma}^{i}, & \text { (d) } D_{\beta}^{\alpha}{ }_{\gamma}=\widetilde{B}_{i}^{\alpha} n_{\beta \gamma}^{i} .
\end{array}
$$

Proof. First, taking into account (2.32a), (2.31b), (2.7d), (2.24b) and (2.29c), we deduce that

$$
\begin{aligned}
\tilde{N}_{i}^{a} n_{\beta}^{i}=\frac{1}{2} \tilde{N}_{i}^{a} \frac{\partial}{\partial v^{\beta}}\left(v^{\gamma} H_{\gamma}^{b} N_{b}^{i}\right) & =\frac{1}{2} \widetilde{N}_{i}^{a}\left\{H_{\beta}^{b} N_{b}^{i}+v^{\gamma} \frac{\partial}{\partial v^{\beta}}\left(H_{\gamma}^{b} N_{b}^{i}\right)\right\} \\
& =\frac{1}{2} H_{\beta}^{a}+\frac{1}{2} H_{\beta}{ }^{a}{ }_{\gamma} v^{\gamma}=H_{\beta}^{a},
\end{aligned}
$$

which proves (2.33a). In a similar way, by using $(2.32 \mathrm{a}),(2.31 \mathrm{~b}),(2.7 \mathrm{~b}),(2.21)$ and (2.15b) we obtain $(2.33 \mathrm{~b})$. Next, contracting $(2.33 \mathrm{a})$ and $(2.33 \mathrm{~b})$ by $N_{a}^{k}$ and $B_{\alpha}^{k}$ 
respectively, and subtracting these equations, we infer that

$$
H_{\beta}^{a} N_{a}^{k}-D_{\beta}^{\alpha} B_{\alpha}^{k}=n_{\beta}^{k},
$$

via (2.7e). Then, taking the derivatives of (2.34) with respect to $v^{\gamma}$ and by using $(2.7 \mathrm{c})$ and $(2.24 \mathrm{~b})$, we obtain

$$
\widetilde{N}_{k}^{b} n_{\beta \gamma}^{k}=\widetilde{N}_{k}^{b} \frac{\partial}{\partial v^{\gamma}}\left(H_{\beta}^{a} N_{a}^{k}\right)-\left(\widetilde{N}_{k}^{b} B_{\alpha}^{k}\right) \frac{\partial D_{\beta}^{\alpha}}{\partial v^{\gamma}}=H_{\beta}^{b}{ }^{b} .
$$

Thus the proof of $(2.33 \mathrm{c})$ is done. Finally, we take again the derivatives of $(2.34)$ with respect to $v^{\gamma}$ and by using $(2.7 \mathrm{a}),(2.7 \mathrm{~b}),(2.13),(2.7 \mathrm{~d})$ and $(2.24 \mathrm{a})$, we deduce that

$$
\begin{aligned}
\widetilde{B}_{k}^{\varepsilon} n_{\beta \gamma}^{k} & =\widetilde{B}_{k}^{\varepsilon} \frac{\partial}{\partial v^{\gamma}}\left(H_{\beta}^{a} N_{a}^{k}\right)-\frac{\partial D_{\beta}^{\varepsilon}}{\partial v^{\gamma}}=-\left(H_{\beta}^{a} N_{a}^{k}\right) \frac{\partial \widetilde{B}_{k}^{\varepsilon}}{\partial v^{\gamma}}-\frac{\partial D_{\beta}^{\varepsilon}}{\partial v^{\gamma}} \\
& =-2 H_{\beta}^{a} N_{a}^{k} g_{b}{ }^{\varepsilon}{ }_{\gamma} \widetilde{N}_{k}^{b}-\frac{\partial D_{\beta}^{\varepsilon}}{\partial v^{\gamma}}=-2 H_{\beta}^{a} g_{a}{ }^{\varepsilon}{ }_{\gamma}-\frac{\partial D_{\beta}^{\varepsilon}}{\partial v^{\gamma}}=D_{\beta}{ }^{\varepsilon}{ }_{\gamma} .
\end{aligned}
$$

This completes the proof of the theorem.

Corollary 2.1. Let $\mathbb{F}^{m}$ be a Finsler submanifold of $\widetilde{\mathbb{F}}^{m+p}$. Then we have:

$$
G_{\beta}^{\alpha} B_{\alpha}^{i}+n_{\beta}^{i}=B_{\beta \gamma}^{i} v^{\gamma}+\widetilde{G}_{j}^{i} B_{\beta}^{j},
$$

$$
G_{\beta}{ }^{\alpha}{ }_{\gamma} B_{\alpha}^{i}+n_{\beta \gamma}^{i}=B_{\beta \gamma}^{i}+\widetilde{G}_{j k}{ }^{i} B_{\beta}^{j} B_{\gamma}^{k} .
$$

Proof. By using (2.34) into (2.14), we obtain (2.35). Then, contracting (2.33a) by $N_{a}^{k}$, and $(2.33 \mathrm{~b})$ by $B_{\alpha}^{k}$, and then adding and using $(2.7 \mathrm{e})$, we deduce that

$$
H_{\beta}{ }^{a}{ }_{\gamma} N_{a}^{k}+D_{\beta}{ }^{\alpha}{ }_{\gamma} B_{\alpha}^{k}=n_{\beta \gamma}^{k} .
$$

Finally, (2.36) is obtained from (2.23) by using (2.37).

Corollary 2.2. Let $\mathbb{F}^{m}$ be a Finsler submanifold of $\widetilde{\mathbb{F}}^{m+p}$. Then we have the assertions:

(i) The canonical horizontal distribution of $\mathbb{F}^{m}$ is locally spanned by the vector fields:

$$
\frac{\delta}{\delta u^{\alpha}}=B_{\alpha}^{i} \frac{\delta}{\delta x^{i}}+n_{\alpha}^{i} \frac{\partial}{\partial y^{i}} .
$$

(ii) The local components of the normal curvature vector field are positively homogeneous of degree 2 with respect to $\left(v^{\beta}\right)$.

Proof. By using (2.20) and (2.34) we obtain (2.38). Then, contracting (2.34) by $v^{\beta}$, and using (2.32a) and $(2.31 \mathrm{~b})$, we deduce that

$$
\frac{\partial n^{k}}{\partial v^{\beta}} v^{\beta}=2 n^{k}
$$

Thus, the assertion (ii) follows from (2.39) by using the Euler Theorem for positively homogeneous functions.

Next, for any $X \in \Gamma(T T M)$ we define the differential operator

$$
\begin{aligned}
& \bar{\nabla}_{X}: \Gamma\left(V T \widetilde{M}_{\mid T M}\right) \rightarrow \Gamma\left(V T \widetilde{M}_{\mid T M}\right), \\
& \bar{\nabla}_{X} \widetilde{Y}=\widetilde{\nabla}_{X} \widetilde{Y}, \forall \widetilde{Y} \in \Gamma\left(V T \widetilde{M}_{\mid T M}\right),
\end{aligned}
$$


where $\widetilde{\nabla}$ is the linear connection from the Berwald connection $\widetilde{B F C}$ of $\widetilde{\mathbb{F}}^{m+p}$ given by $(2.22)$. Therefore $\overline{B F C}=(H T M, \bar{\nabla})$ is a vectorial Finsler connection on $\mathbb{F}^{m}$ with respect to the vector bundle $V T \widetilde{M}_{\mid T M}$ (see Bejancu [5], p. 26). By (2.40) and (2.22) we deduce that $\bar{\nabla}$ is locally given by

$$
\begin{gathered}
\text { (a) } \bar{\nabla}_{\frac{\delta}{\delta u^{\gamma}}} \frac{\partial}{\partial y^{j}}=\bar{G}_{j}{ }^{i} \gamma \frac{\partial}{\partial y^{i}}, \quad \text { (b) } \bar{G}_{j}{ }^{i}{ }_{\gamma}=\widetilde{G}_{j}{ }^{i}{ }_{k} B_{\gamma}^{k}, \\
\text { (c) } \bar{\nabla}_{\frac{\partial}{\partial v^{\gamma}}} \frac{\partial}{\partial y^{j}}=0 .
\end{gathered}
$$

Also, we note that $B_{\alpha}^{i}, \alpha \in\{1, \ldots, m\}$ and $i \in\{1, \ldots, m+p\}$ are the local components of a mixed Finsler tensor field on $\mathbb{F}^{m}$ with respect to the vector bundle $V T \widetilde{M}_{\mid T M}$. Now, we can state the following important result.

Theorem 2.2. Let $\mathbb{F}^{m}$ be a Finsler submanifold of $\widetilde{\mathbb{F}}^{m+p}$. Then the horizontal relative covariant derivative of $B_{\alpha}^{i}$ with respect to the pair $(\overline{B F C}, B F C)$ is completely determined by the normal curvature vector field $\mathbf{n}$ of $\mathbb{F}^{m}$ as follows:

$$
B_{\alpha \mid \beta}^{i}=n_{\alpha \beta}^{i}
$$

Proof. By using the local formulae for the horizontal relative covariant derivative (cf. Bejancu-Farran [7], p. 29) and taking into account (2.41b) and (2.36), we obtain

$$
\begin{aligned}
B_{\alpha \mid \beta}^{i} & =B_{\alpha \beta}^{i}+B_{\alpha}^{j} \bar{G}_{j}{ }^{i}{ }_{\beta}-G_{\alpha}{ }^{\varepsilon}{ }_{\beta} B_{\varepsilon}^{i} \\
& =B_{\alpha \beta}^{i}+\widetilde{G}_{j}{ }^{i}{ }_{k} B_{\alpha}^{j} B_{\beta}^{k}-G_{\alpha}{ }^{\varepsilon}{ }_{\beta} B_{\varepsilon}^{i}=n_{\alpha \beta}^{i} .
\end{aligned}
$$

Thus the proof is done.

Finally, by using $(2.41 \mathrm{~b}),(2.38)$ and $(1.11)$, we deduce that

$$
\begin{aligned}
\widetilde{g}_{i j \mid \alpha} & =\frac{\delta \widetilde{g}_{i j}}{\delta u^{\alpha}}-\widetilde{g}_{h j} \bar{G}_{i}{ }^{h}{ }_{\alpha}-\widetilde{g}_{i h} \bar{G}_{j}{ }^{h}{ } \\
& =\left(\frac{\delta \widetilde{g}_{i j}}{\delta x^{k}}-\widetilde{g}_{h j} \widetilde{G}_{i}{ }^{h}{ }_{k}-\widetilde{g}_{i h} \widetilde{G}_{j}{ }^{h}{ }_{k}\right) B_{\alpha}^{k}+n_{\alpha}^{k} \frac{\partial \widetilde{g}_{i j}}{\partial y^{k}} \\
& =\widetilde{g}_{i j \mid k} B_{\alpha}^{k}+2 n_{\alpha}^{k} \widetilde{g}_{i j k} .
\end{aligned}
$$

\section{Structure Equations and Curvature of a Finsler Submanifold}

The purpose of this section is to obtain the structure equations of the immersion of $\mathbb{F}^{m}$ in $\widetilde{\mathbb{F}}^{m+p}$. More precisely, we want to relate $R^{\alpha}{ }_{\beta \gamma}$ with $\widetilde{R}_{j k}^{i}$ and $\left\{H_{\alpha}{ }^{\varepsilon}{ }_{\beta \gamma}, G_{\alpha}{ }^{\varepsilon} \beta \gamma\right\}$ with $\left\{\widetilde{H}_{j}{ }^{i}{ }_{k j}, \widetilde{G}_{j}{ }^{i} k h\right\}$. This will enable us to relate the flag curvatures of $\mathbb{F}^{m}$ and $\widetilde{\mathbb{F}}^{m+p}$.

First, we consider the associate linear connection $\widetilde{D}$ to the Berwald connection $\widetilde{B F C}=(H T \widetilde{M}, \widetilde{\nabla})=\left(\widetilde{G}_{j}^{i}, \widetilde{G}_{j}{ }^{i}{ }_{k}, 0\right)$ given by (cf. Bejancu-Farran [7], p. 30)

$$
\widetilde{D}_{X} Y=\widetilde{\nabla}_{X} \widetilde{v} Y+\widetilde{Q} \widetilde{\nabla}_{X} \widetilde{Q} \widetilde{h} Y, \quad \forall X, Y \in \Gamma(T T \widetilde{M}),
$$

where $\widetilde{h}$ and $\widetilde{v}$ are the projection morphisms of $T T \widetilde{M}$ on $H T \widetilde{M}$ and $V T \widetilde{M}$ respectively, and $\widetilde{Q}$ is the Finsler almost product structure on $T \widetilde{M}$ given by

$$
\widetilde{Q}\left(\frac{\delta}{\delta x^{i}}\right)=\frac{\partial}{\partial y^{i}}, \quad \widetilde{Q}\left(\frac{\partial}{\partial y^{i}}\right)=\frac{\delta}{\delta x^{i}} .
$$


Then, by using (3.1), (3.2) and (2.22), we obtain
(a) $\widetilde{D}_{\frac{\delta}{\delta x^{k}}} \frac{\delta}{\delta x^{j}}=\widetilde{G}_{j k}{ }^{i} \frac{\delta}{\delta x^{i}}$
(b) $\widetilde{D}_{\frac{\delta}{\delta x^{k}}} \frac{\partial}{\partial y^{j}}=\widetilde{G}_{j}{ }^{i} k \frac{\partial}{\partial y^{i}}$,
(c) $\widetilde{D}_{\frac{\partial}{\partial y^{k}}} \frac{\delta}{\delta x^{j}}=0$,
(d) $\widetilde{D}_{\frac{\partial}{\partial y^{k}}} \frac{\partial}{\partial y^{j}}=0$.

Taking into account (2.38), (3.3) and (2.41b) we deduce that

$$
\widetilde{D}_{\frac{\delta}{\delta u^{\gamma}}} \frac{\delta}{\delta u^{\beta}}=\left(B_{\beta \gamma}^{i}+\widetilde{G}_{j k}{ }^{i} B_{\beta}{ }^{j} B_{\gamma}^{k}\right) \frac{\delta}{\delta x^{i}}+\left(\frac{\delta n_{\beta}^{i}}{\delta u^{\gamma}}+n_{\beta}^{j} \bar{G}_{j}{ }^{i} \gamma\right) \frac{\partial}{\partial y^{i}} .
$$

Next, we denote by $\widetilde{T}$ the torsion tensor field of $\widetilde{D}$ and by using (3.4) and (1.10a) we infer that

$$
\begin{aligned}
& \widetilde{T}\left(\frac{\delta}{\delta u^{\gamma}}, \frac{\delta}{\delta u^{\beta}}\right)=\widetilde{D}_{\frac{\delta}{\delta u^{\gamma}}} \frac{\delta}{\delta u^{\beta}}-\widetilde{D}_{\frac{\delta}{\delta u^{\beta}}} \frac{\delta}{\delta u^{\gamma}}-\left[\frac{\delta}{\delta u^{\gamma}}, \frac{\delta}{\delta u^{\beta}}\right] \\
& =\left\{\left(\frac{\delta n_{\beta}^{i}}{\delta u^{\gamma}}+n_{\beta}^{j} \bar{G}_{j}{ }^{i}{ }_{\gamma}-n_{\alpha}^{i} G_{\beta}{ }^{\alpha}{ }_{\gamma}\right)-\left(\frac{\delta n_{\gamma}^{i}}{\delta u^{\beta}}+n_{\gamma}^{j} \bar{G}_{j}{ }^{i}{ }_{\beta}-n_{\alpha}^{i} G_{\gamma}{ }^{\alpha}{ }_{\beta}\right)\right\} \frac{\partial}{\partial y^{i}} \\
& -R^{\alpha \beta} \frac{\partial}{\partial v^{\alpha}}=\left(R^{\alpha}{ }_{\beta \gamma} B_{\alpha}^{i}+n_{\beta \mid \gamma}^{i}-n_{\gamma \mid \beta}^{i}\right) \frac{\partial}{\partial y^{i}} .
\end{aligned}
$$

On the other hand, by using (1.10) for $\widetilde{\mathbb{F}}^{m+p}$ and (3.3) we deduce that

$$
\widetilde{T}\left(\frac{\delta}{\delta x^{k}}, \frac{\delta}{\delta x^{j}}\right)=\widetilde{R}_{j k}^{i} \frac{\partial}{\partial y^{i}} \text { and } \widetilde{T}\left(\frac{\delta}{\delta x^{k}}, \frac{\partial}{\partial y^{j}}\right)=\widetilde{T}\left(\frac{\partial}{\partial y^{k}}, \frac{\partial}{\partial y^{j}}\right)=0 .
$$

Hence we have

$$
\begin{aligned}
\widetilde{T}\left(\frac{\delta}{\delta u^{\gamma}}, \frac{\delta}{\delta u^{\beta}}\right) & =\widetilde{T}\left(B_{\gamma}^{k} \frac{\delta}{\delta x^{k}}+n_{\gamma}^{k} \frac{\partial}{\partial y^{k}}, B_{\beta}^{j} \frac{\delta}{\delta x^{j}}+n_{\beta}^{j} \frac{\partial}{\partial y^{j}}\right) \\
& =\widetilde{T}\left(\frac{\delta}{\delta x^{k}}, \frac{\delta}{\delta x^{j}}\right) B_{\beta}^{j} B_{\gamma}^{k}=\widetilde{R}^{i}{ }_{j k} B_{\beta}^{j} B_{\gamma}^{k} \frac{\partial}{\partial y^{i}} .
\end{aligned}
$$

Comparing (3.5) and (3.6) we can state the following.

Theorem 3.1. Let $\mathbb{F}^{m}$ be a Finsler submanifold of $\widetilde{\mathbb{F}}^{m+p}$. Then we have:

$$
\widetilde{R}_{j k}^{i} B_{\beta}^{j} B_{\gamma}^{k}=R_{\beta \gamma}^{\alpha} B_{\alpha}^{i}+n_{\beta \mid \gamma}^{i}-n_{\gamma \mid \beta}^{i},
$$

where "I stands for the horizontal relative covariant derivative with respect to the pair $(\overline{B F C}, B F C)$.

Remark 3.1. By similar calculations for $\widetilde{T}\left(\delta / \delta u^{\gamma}, \partial / \partial v^{\beta}\right)$ and $\widetilde{T}\left(\partial / \partial v^{\gamma}, \partial / \partial v^{\beta}\right)$ we obtain (2.36) and a trivial identity, respectively.

Next, by using (2.38), (2.4b), (2.22) and (2.36) we obtain
(a) $\widetilde{\nabla}_{\frac{\delta}{\delta u \beta}} \frac{\partial}{\partial v^{\alpha}}=\left(B_{\alpha \beta}^{i}+\widetilde{G}_{j}{ }^{i}{ }_{k} B_{\alpha}^{j} B_{\beta}^{k}\right) \frac{\partial}{\partial y^{i}}=\left(G_{\alpha}{ }^{\varepsilon}{ }_{\beta} B_{\varepsilon}^{i}+n_{\alpha \beta}^{i}\right) \frac{\partial}{\partial y^{i}}$,
(b) $\widetilde{\nabla}_{\frac{\partial}{\partial v \beta}} \frac{\partial}{\partial v^{\alpha}}=0$. 
Taking into account (2.38), (2.22) and the first equality in (3.8a) we deduce that

$$
\begin{aligned}
& \widetilde{\nabla}_{\frac{\delta}{\delta u^{\gamma}}} \widetilde{\nabla}_{\frac{\delta}{\delta u^{\beta}}} \frac{\partial}{\partial v^{\alpha}}=\widetilde{\nabla}_{\frac{\delta}{\delta u^{\gamma}}}\left(B_{\alpha \beta}^{i}+\widetilde{G}_{j}{ }^{i}{ }_{k} B_{\alpha}^{j} B_{\beta}^{k}\right) \frac{\partial}{\partial y^{i}} \\
& =\left(B_{\alpha \beta \gamma}^{i}+\frac{\delta}{\delta u^{\gamma}}\left(\widetilde{G}_{j}{ }^{i}{ }_{k} B_{\alpha}^{j} B_{\beta}^{k}\right)+\widetilde{G}_{j}{ }^{i}{ }_{k} B_{\alpha \beta}^{j} B_{\gamma}^{k}+\widetilde{G}_{j}{ }_{k}{ }_{k} \widetilde{G}_{t}{ }^{i}{ }_{h} B_{\alpha}^{j} B_{\beta}^{k} B_{\gamma}^{h}\right) \frac{\partial}{\partial y^{i}} .
\end{aligned}
$$

On the other hand, by direct calculations using (1.8b) for $\widetilde{\mathbb{F}}^{m+p}$ and (2.38) we obtain

$$
\frac{\delta}{\delta u^{\gamma}}\left(\widetilde{G}_{j}{ }^{i}{ }_{k} B_{\alpha}^{j} B_{\beta}^{k}\right)=\frac{\delta \widetilde{G}_{j}{ }^{i} k}{\delta x^{h}} B_{\alpha}^{j} B_{\beta}^{k} B_{\gamma}^{h}+\widetilde{G}_{j k h}{ }^{i} B_{\alpha}^{j} B_{\beta}^{k} n_{\gamma}^{h}+\widetilde{G}_{j}{ }^{i}{ }_{k} B_{\alpha \gamma}^{i} B_{\beta}^{k}+\widetilde{G}_{j k}{ }^{i}{ } B_{\alpha}^{j} B_{\beta \gamma}^{k} .
$$

Thus we have

$$
\begin{aligned}
& \widetilde{\nabla}_{\frac{\delta}{\delta u^{\gamma}}} \widetilde{\nabla}_{\frac{\delta}{\delta u^{\beta}}} \frac{\partial}{\partial v^{\alpha}}=\left\{B_{\alpha \beta \gamma}^{i}+\left(\frac{\delta \widetilde{G}_{j k}{ }^{i} k}{\delta x^{h}}+\widetilde{G}_{j}{ }^{t}{ }_{k} \widetilde{G}_{t}{ }^{i} h\right) B_{\alpha}^{j} B_{\beta}^{k} B_{\gamma}^{h}\right. \\
+ & \left.\widetilde{G}_{j k h}{ }^{i}{ }_{k h}{ }^{j} B_{\beta}^{k} n_{\gamma}^{h}+\widetilde{G}_{j}{ }^{i}{ }_{k} B_{\alpha \gamma}^{i} B_{\beta}^{k}+\widetilde{G}_{j}{ }^{i}{ }_{k} B_{\alpha}^{j} B_{\beta \gamma}^{k}+\widetilde{G}_{j}{ }^{i}{ }_{k} B_{\alpha \beta}^{j} B_{\gamma}^{k}\right\} \frac{\partial}{\partial y^{i}} .
\end{aligned}
$$

Also, by using (1.10a) and (3.8b) we infer that

$$
\widetilde{\nabla}_{\left[\frac{\delta}{\delta u^{\gamma}}, \frac{\delta}{\delta u^{\beta}}\right]} \frac{\partial}{\partial v^{\alpha}}=0 .
$$

Then, we denote by $\widetilde{R}$ the curvature tensor field of the linear connection $\widetilde{\nabla}$ from the Berwald connection $\widetilde{B F C}=(H T \widetilde{M}, \widetilde{\nabla})$ on $\widetilde{\mathbb{F}}^{m+p}$, and by using the first equality in $(1.8 \mathrm{a})$ for $\widetilde{\mathbb{F}}^{m+p},(3.9)$ and $(3.10)$ we obtain

$$
\begin{aligned}
& \widetilde{R}\left(\frac{\delta}{\delta u^{\gamma}}, \frac{\delta}{\delta u^{\beta}}\right) \frac{\partial}{\partial v^{\alpha}} \\
& \quad=\widetilde{\nabla}_{\frac{\delta}{\delta u^{\gamma}}} \widetilde{\nabla}_{\frac{\delta}{\delta u^{\beta}}} \frac{\partial}{\partial v^{\alpha}}-\widetilde{\nabla}_{\frac{\delta}{\delta u^{\beta}}} \widetilde{\nabla}_{\frac{\delta}{\delta u^{\gamma}}} \frac{\partial}{\partial v^{\alpha}}-\widetilde{\nabla}_{\left[\frac{\delta}{\delta u^{\gamma}}, \frac{\delta}{\delta u^{\beta}}\right]} \frac{\partial}{\partial v^{\alpha}} \\
& \quad=\left\{\widetilde{H}_{j}{ }^{i} k h B_{\alpha}^{j} B_{\beta}^{k} B_{\gamma}^{h}+\widetilde{G}_{j k h}{ }^{i}{ }_{k h} B_{\alpha}^{j}\left(B_{\beta}^{k} n_{\gamma}^{h}-B_{\gamma}^{k} n_{\beta}^{h}\right\} .\right.
\end{aligned}
$$

On the other hand, taking into account the second equality in (3.8a) and using (2.41) and (2.36) we deduce that

$$
\begin{aligned}
& \widetilde{\nabla}_{\frac{\delta}{\delta u^{\gamma}}} \widetilde{\nabla}_{\frac{\delta}{\delta u^{\beta}}} \frac{\partial}{\partial v^{\alpha}}=\frac{\delta}{\delta u^{\gamma}}\left(G_{\alpha}{ }^{\varepsilon} B_{\varepsilon}^{i}+n_{\alpha \beta}^{i}\right) \frac{\partial}{\partial y^{i}}+\left(G_{\alpha}{ }^{\varepsilon}{ }_{\beta} B_{\varepsilon}^{i}+n_{\alpha \beta}^{i}\right) \bar{\nabla}_{\frac{\delta}{\delta u^{\gamma}}} \frac{\partial}{\partial y^{i}} \\
& =\left\{\frac{\delta G_{\alpha}{ }^{\varepsilon} \beta}{\delta u^{\gamma}} B_{\varepsilon}^{i}+G_{\alpha}{ }^{\varepsilon}{ }_{\beta} B_{\varepsilon \gamma}^{i}+\frac{\delta n_{\alpha \beta}^{i}}{\delta u^{\gamma}}+G_{\alpha}{ }^{\varepsilon}{ }_{\beta} B_{\varepsilon}^{j} \widetilde{G}_{j}{ }^{i}{ }_{k} B_{\gamma}^{k}+n_{\alpha \beta}^{j} \bar{G}_{j}{ }^{i} \gamma\right\} \frac{\partial}{\partial y^{i}} \\
& =\left\{\frac{\delta G_{\alpha}{ }^{\beta} \beta}{\delta u^{\gamma}} B_{\varepsilon}^{i}+G_{\alpha}{ }^{\varepsilon}{ }_{\beta}\left(B_{\varepsilon \gamma}^{i}+\widetilde{G}_{j}{ }^{i}{ }_{k} B_{\varepsilon}^{j} B_{\gamma}^{k}\right)+\frac{\delta n_{\alpha \beta}^{i}}{\delta u^{\gamma}}+n_{\alpha \beta}^{j} \bar{G}_{j}{ }^{i} \gamma\right\} \frac{\partial}{\partial y^{i}} \\
& =\left\{\left(\frac{\delta G_{\alpha}{ }^{\varepsilon} \beta}{\delta u^{\gamma}}+G_{\alpha}{ }^{\mu}{ }_{\beta} G_{\mu}{ }^{\varepsilon} \gamma\right) B_{\varepsilon}^{i}+n_{\varepsilon \gamma}^{i} G_{\alpha} \varepsilon_{\beta}+\frac{\delta n_{\alpha \beta}^{i}}{\delta u^{\gamma}}+n_{\alpha \beta}^{j} \bar{G}_{j}^{i} \gamma\right\} \frac{\partial}{\partial y^{i}} .
\end{aligned}
$$


Finally, by a similar calculation as in (3.11), but by using (3.12), (3.10) and (1.8), we obtain

$$
\widetilde{R}\left(\frac{\delta}{\delta u^{\gamma}}, \frac{\delta}{\delta u^{\beta}}\right) \frac{\partial}{\partial v^{\alpha}}=\left\{H_{\alpha}{ }^{\varepsilon}{ }_{\beta \gamma} B_{\varepsilon}^{i}+n_{\alpha \beta \mid \gamma}^{i}-n_{\alpha \gamma \mid \beta}^{i}\right\} \frac{\partial}{\partial y^{i}} .
$$

Thus, comparing (3.11) with (3.13) we deduce that

$$
\widetilde{H}_{j k h}{ }^{i} B_{\alpha}^{j} B_{\beta}^{k} B_{\gamma}^{h}+\widetilde{G}_{j}{ }^{i} k h B_{\alpha}^{j}\left(B_{\beta}^{k} n_{\gamma}^{h}-B_{\gamma}^{k} n_{\beta}^{h}\right)=H_{\alpha \beta \gamma}{ }^{\varepsilon} B_{\varepsilon}^{i}+n_{\alpha \beta \mid \gamma}^{i}-n_{\alpha \gamma \mid \beta}^{i} .
$$

Next, by using the first equality in (3.8a) and taking into account (1.8b) for $\widetilde{\mathbb{F}}^{m+p},(2.4 \mathrm{~b})$ and $(2.41 \mathrm{c})$ we infer that

$$
\widetilde{\nabla}_{\frac{\partial}{\partial v^{\gamma}}} \widetilde{\nabla}_{\frac{\delta}{\delta u \beta}} \frac{\partial}{\partial v^{\alpha}}=\widetilde{G}_{j k h}{ }^{i} B_{\alpha}^{j} B_{\beta}^{k} B_{\gamma}^{h} \frac{\partial}{\partial y^{i}} .
$$

On the other hand, by using the second equality in (3.8a) and taking into account (1.8b), (2.41c) and (2.32c) we obtain

$$
\widetilde{\nabla}_{\frac{\partial}{\partial v^{\gamma}}} \widetilde{\nabla}_{\frac{\delta}{\delta u \beta}} \frac{\partial}{\partial v^{\alpha}}=\left(G_{\alpha}{ }^{\varepsilon}{ }_{\beta \gamma} B_{\varepsilon}^{i}+n_{\alpha \beta \gamma}^{i}\right) \frac{\partial}{\partial y^{i}} .
$$

Comparing these equalities we deduce that

$$
\widetilde{G}_{j k h}{ }^{i} B_{\alpha}^{i} B_{\beta}^{k} B_{\gamma}^{h}=G_{\alpha}{ }^{\varepsilon} \beta \gamma B_{\varepsilon}^{i}+n_{\alpha \beta \gamma}^{i} .
$$

Now, we are able to state the following.

Theorem 3.2. Let $\mathbb{F}^{m}$ be a Finsler submanifold of $\widetilde{\mathbb{F}}^{m+p}$. Then the curvature tensor fields of Berwald connections on $\mathbb{F}^{m}$ and $\widetilde{\mathbb{F}}^{m+p}$ are related by the following structure equations:

(a) $\widetilde{H}_{j t k h} B_{\alpha}^{j} B_{\mu}^{t} B_{\beta}^{k} B_{\gamma}^{h}+\widetilde{G}_{j t k h} B_{\alpha}^{j} B_{\mu}^{t}\left(B_{\beta}^{k} n_{\gamma}^{h}-B_{\gamma}^{k} n_{\beta}^{h}\right)$

$$
=H_{\alpha \mu \beta \gamma}+\tilde{g}_{i t}\left(n_{\alpha \beta \mid \gamma}^{i}-n_{\alpha \gamma \mid \beta}^{i}\right) B_{\mu}^{t},
$$

(b) $\widetilde{G}_{j t k h} B_{\alpha}^{j} B_{\mu}^{t} B_{\beta}^{k} B_{\gamma}^{h}=G_{\alpha \mu \beta \gamma}+\widetilde{g}_{i t} n_{\alpha \beta \gamma}^{i} B_{\mu}^{t}$.

and

$$
\text { (a) } \begin{array}{r}
\widetilde{H}_{j t k h} B_{\alpha}^{j} N_{a}^{t} B_{\beta}^{k} B_{\gamma}^{h}+\widetilde{G}_{j t k h} B_{\alpha}^{j} N_{a}^{t}\left(B_{\beta}^{k} n_{\gamma}^{h}-B_{\gamma}^{k} n_{\beta}^{h}\right) \\
=\widetilde{g}_{i t}\left(n_{\alpha \beta \mid \gamma}^{i}-n_{\alpha \gamma \mid \beta}^{i}\right) N_{a}^{t},
\end{array}
$$

(b) $\widetilde{G}_{j t k h} B_{\alpha}^{j} N_{a}^{t} B_{\beta}^{k} B_{\gamma}^{h}=\widetilde{g}_{i t} n_{\alpha \beta \gamma}^{i} N_{a}^{t}$.

Proof. By contracting (3.14) by $\widetilde{g}_{i t} B_{\mu}^{t}$ and $\widetilde{g}_{i t} N_{a}^{t}$ we obtain (3.16a) and (3.17a). In a similar way we obtain (3.16b) and (3.17b) from (3.15).

According to the terminology from the theory of Riemannian submanifolds, we call (3.16) and (3.17) the Gauss-Berwald equations and the Codazzi-Berwald equations for the immersion of $\mathbb{F}^{m}$ into $\widetilde{\mathbb{F}}^{m+p}$.

Now, we want to relate the flag curvatures of $\mathbb{F}^{m}$ and $\widetilde{\mathbb{F}}^{m+p}$. To this end we start with a contraction of (3.7) by $\widetilde{g}_{i h} v^{\beta}$ and by using (1.16) for both $\mathbb{F}^{m}$ and $\widetilde{\mathbb{F}}^{m+p}$ and (2.2) we obtain

$$
\widetilde{R}_{h k} B_{\gamma}^{k}=R_{\mu \gamma} \widetilde{B}_{h}^{\mu}+\widetilde{g}_{i h}\left(n_{\beta \mid \gamma}^{i}-n_{\gamma \mid \beta}^{i}\right) v^{\beta} .
$$

Contracting this by $B_{\alpha}^{h}$ and using (2.7a) we deduce that

$$
\widetilde{R}_{h k} B_{\alpha}^{h} B_{\gamma}^{k}=R_{\alpha \gamma}+\widetilde{g}_{i h}\left(n_{\beta \mid \gamma}^{i}-n_{\gamma \mid \beta}^{i}\right) B_{\alpha}^{h} v^{\beta} .
$$


Theorem 3.3. Let $\mathbb{F}^{m}=(M, F)$ be a Finsler submanifold of $\widetilde{\mathbb{F}}^{m+p}=(\widetilde{M}, \widetilde{F})$, and $X=\left(X^{\gamma}\right)$ be a tangent vector to $M$ at the point $u \in M$. Then the flag curvatures of $\mathbb{F}^{m}$ and $\widetilde{\mathbb{F}}^{m+p}$ at the point $u$ with respect to the flag $\Pi(X)$ are related by

$$
K(X)=\widetilde{K}(X)+\frac{\widetilde{g}_{i h}\left(n_{\gamma \mid \beta}^{i}-n_{\beta \mid \gamma}^{i}\right) B_{\alpha}^{h} v^{\beta} X^{\alpha} X^{\gamma}}{F^{2} h_{\alpha \gamma} X^{\alpha} X^{\gamma}} .
$$

Proof. Contracting (3.18) by $X^{\alpha} X^{\gamma}$ and setting $\tilde{X}^{i}=B_{\alpha}^{i} X^{\alpha}$, we deduce that

$$
\widetilde{R}_{h k} \widetilde{X}^{h} \widetilde{X}^{k}=R_{\alpha \gamma} X^{\alpha} X^{\gamma}+\widetilde{g}_{i h}\left(n_{\beta \mid \gamma}^{i}-n_{\gamma \mid \beta}^{i}\right) v^{\beta} B_{\alpha}^{h} X^{\alpha} X^{\gamma} .
$$

If $\widetilde{X}=\left(\tilde{X}^{i}\right)$, then it is easy to check that

$$
g(X, X)=\widetilde{g}(\tilde{X}, \tilde{X}), g(v, X)=\widetilde{g}(y, \widetilde{X}) \text { and } g(u, v)=\widetilde{g}(y, y) .
$$

Therefore, $\Delta(X)$ from $(1.18)$ is the same with $\Delta(\tilde{X})$ for $\widetilde{\mathbb{F}}^{m+p}$. Then, dividing (3.20) by $\Delta(X)$ and taking into account (1.17) for both $\mathbb{F}^{m}$ and $\widetilde{\mathbb{F}}^{m+p}$, we obtain (3.19)

From (3.19) we see that the flag curvatures of $\mathbb{F}^{m}$ and $\widetilde{\mathbb{F}}^{m+p}$ are related by means of the relative horizontal covariant derivatives of $n_{\alpha}^{i}$ with respect to the pair $(\overline{B F C}, B F C)$. However, according to the theory of Riemannian submanifolds, the second fundamental forms of $\mathbb{F}^{m}$ (defined in Section 2) should be involved in (3.19). To show this, we examine the last term in (3.19).

First, by using (2.8a), (2.34), (2.7a) and (2.7b), we obtain

$$
\widetilde{g}_{i h} n_{\beta}^{i} B_{\alpha}^{h}=g_{\alpha \varepsilon} \widetilde{B}_{i}^{\varepsilon} n_{\beta}^{i}=g_{\alpha \varepsilon} \widetilde{B}_{i}^{\varepsilon}\left(H_{\beta}^{a} N_{a}^{i}-D_{\beta}^{\mu} B_{\mu}^{i}\right)=-g_{\alpha \varepsilon} D_{\beta}^{\varepsilon}=-D_{\alpha \beta} .
$$

Taking the relative horizontal covariant derivatives of this equality, and using (2.43), we obtain

$$
\widetilde{g}_{i h} n_{\beta \mid \gamma}^{i} B_{\alpha}^{h}=-D_{\alpha \beta \mid \gamma}-\widetilde{g}_{i h \mid k} n_{\beta}^{i} B_{\alpha}^{h} B_{\gamma}^{k}-2 \widetilde{g}_{i h k} n_{\beta}^{i} B_{\alpha}^{h} n_{\gamma}^{k}-\widetilde{g}_{i h} n_{\beta}^{i} B_{\alpha \mid \gamma}^{h} .
$$

On the other hand, by using (2.34), (2.37), (2.6) and (2.3), we infer that

$$
\widetilde{g}_{i h} n_{\beta}^{i} B_{\alpha \mid \gamma}^{h}=\widetilde{g}_{i h}\left(H_{\beta}^{a} N_{a}^{i}-D_{\beta}^{\mu} B_{\mu}^{i}\right)\left(H_{\alpha}{ }^{b}{ }_{\gamma} N_{b}^{h}+D_{\alpha}{ }^{\varepsilon}{ }_{\gamma} B_{\varepsilon}^{h}\right)=H_{a \alpha \gamma} H_{\beta}^{a}-D_{\alpha}{ }^{\varepsilon}{ }_{\gamma} D_{\varepsilon \beta} .
$$

Thus we have

$$
\widetilde{g}_{i h} n_{\beta \mid \gamma}^{i} B_{\alpha}^{h}=D_{\alpha}{ }^{\varepsilon}{ }_{\gamma} D_{\varepsilon \beta}-D_{\alpha \beta \mid \gamma}-H_{a \alpha \gamma} H_{\beta}^{a}-\widetilde{g}_{i h \mid k} n_{\beta}^{i} B_{\alpha}^{h} B_{\gamma}^{k}-2 \widetilde{g}_{i h k} n_{\beta}^{i} B_{\alpha}^{h} n_{\gamma}^{k} .
$$

By using this equation and taking into account that $\widetilde{g}_{i h k}$ is a symmetric Finsler tensor field, we deduce that

$$
\begin{aligned}
\widetilde{g}_{i h}\left(n_{\gamma \mid \beta}^{i}-n_{\beta \mid \gamma}^{i}\right) B_{\alpha}^{h} & =D_{\alpha}{ }^{\varepsilon}{ }_{\beta} D_{\varepsilon \gamma}-D_{\alpha}{ }^{\varepsilon}{ }_{\gamma} D_{\varepsilon \beta}+D_{\alpha \beta \mid \gamma}-D_{\alpha \gamma \mid \beta} \\
& +H_{a \alpha \gamma} H_{\beta}^{a}-H_{a \alpha \beta} H_{\gamma}^{a}+\widetilde{g}_{i h \mid k}\left(n_{\beta}^{i} B_{\gamma}^{k}-n_{\gamma}^{i} B_{\beta}^{k}\right) B_{\alpha}^{h} .
\end{aligned}
$$

Next, by using the last equality in $(2.29 \mathrm{~b})$ and taking into account that $v^{\beta}{ }_{\mid \gamma}=0$, we obtain

$$
D_{\alpha \beta \mid \gamma} v^{\beta}=0
$$

Also, we have

$$
\widetilde{g}_{i h \mid k} B_{\beta}^{k} v^{\beta}=\widetilde{g}_{i h \mid k} y^{k}=0,
$$

since the horizontal covariant derivative of $\widetilde{g}_{i h}$ with respect to Berwald connection in the direction of the supporting element vanishes (cf. Matsumoto [12], p. 119). 
Now, contracting (3.21) by $v^{\beta}$ and using (3.22), (3.23), (2.29), (2.30) and (2.39), we deduce that

$$
\begin{aligned}
\widetilde{g}_{i h}\left(n_{\gamma \mid \beta}^{i}-n_{\beta \mid \gamma}^{i}\right) B_{\alpha}^{h} v^{\beta} & =H_{0}^{a} H_{a \alpha \gamma}-H_{a \alpha} H_{\gamma}^{a}-D_{\alpha \gamma \mid \beta} v^{\beta} \\
& -D_{\alpha}^{\varepsilon} D_{\varepsilon \gamma}+2 \widetilde{g}_{i h \mid k} n^{i} B_{\alpha}^{h} B_{\gamma}^{k} .
\end{aligned}
$$

Finally, by using (3.24) into (3.19), we obtain

$$
\begin{aligned}
K(X) & =\widetilde{K}(X)+\frac{1}{F^{2} h_{\alpha \gamma} X^{\alpha} X^{\gamma}}\left\{H_{0}^{a} H_{a \alpha \gamma}-H_{a \alpha} H_{\gamma}^{a}-D_{\alpha \gamma \mid \beta} v^{\beta}\right. \\
& \left.-D_{\alpha}^{\varepsilon} D_{\varepsilon \gamma}+2 \widetilde{g}_{i h \mid k} n^{i} B_{\alpha}^{h} B_{\gamma}^{k}\right\} X^{\alpha} X^{\gamma} .
\end{aligned}
$$

We close the paper with some applications of (3.25) to some special Finsler immersions. First, we recall that $\mathbb{F}^{m}$ is totally geodesic immersed in $\widetilde{\mathbb{F}}^{m+p}$ if and only if any geodesic of $\mathbb{F}^{m}$ is a geodesic of $\widetilde{\mathbb{F}}^{m+p}$. To reach our goal, we need the following.

Theorem 3.4. (Bejancu [4]) $\mathbb{F}^{m}$ is totally geodesic immersed in $\widetilde{\mathbb{F}}^{m+p}$, if and only if, the normal curvatures of $\mathbb{F}^{m}$ vanish, that is, we have

$$
H_{0}^{a}=0, \quad \forall a \in\{m+1, \ldots, m+p\} .
$$

This enables us to state the following.

Theorem 3.5. $F^{m}$ is totally geodesic immersed in $\widetilde{\mathbb{F}}^{m+p}$, if and only if, both the normal and tangent second fundamental forms vanish, that is, we have

$$
\begin{aligned}
& \text { (a) } H_{\alpha}{ }^{a}{ }_{\beta}=0, \quad \text { and (b) } D_{\alpha}{ }^{\gamma}=0, \\
& \forall \alpha, \beta, \gamma \in\{1, \ldots, m\}, \forall a \in\{m+1, \ldots, m+p\} .
\end{aligned}
$$

Proof. Suppose that $\mathbb{F}^{m}$ is a totally geodesic Finsler submanifold. Then by (3.26) and $(2.31 \mathrm{~b})$ we deduce that $n^{i}=0$, for all $i \in\{1, \ldots, m+p\}$. Thus, taking into account $(2.33 \mathrm{c}),(2.33 \mathrm{~d})$ and $(2.32 \mathrm{~b})$, we obtain $(3.27)$. The converse is a simple consequence of (2.30) via (3.27a) and (3.26).

Theorem 3.6. Let $\mathbb{F}^{m}$ be a totally geodesic Finsler submanifold of $\widetilde{\mathbb{F}}^{m+p}$. Then $\mathbb{F}^{m}$ and $\widetilde{\mathbb{F}}^{m+p}$ have the same flag curvature.

Proof. As $\mathbb{F}^{m}$ is totally geodesic, by (3.27a) and (2.29b) we deduce that

$$
H_{\alpha}^{a}=0, \quad \forall \alpha \in\{1, \ldots, m\}, a \in\{m+1, \ldots, m+p\} .
$$

Also, by using (3.27a) and (2.29a) we obtain

$$
D_{\alpha \beta}=0, \quad \forall \alpha, \beta \in\{1, \ldots, m\} .
$$

Finally, by using (3.28), (3.29), (3.26) and taking into account that $n^{i}=0$ for all $i \in\{1, \ldots, m+p\}$, from $(3.25)$ we infer that

$$
K(X)=\widetilde{K}(X), \quad \forall X \in \Gamma(T M) .
$$

Thus the proof is done.

In particular, we obtain an extension to Finsler geometry of a well known result from Riemannian geometry.

Corollary 3.1. Any totally geodesic Finsler submanifold $\mathbb{F}^{m}$ of a Finsler manifold $\widetilde{\mathbb{F}}^{m+p}$ of constant flag curvature $K$ is of constant flag curvature $K$, too. 
Next, we suppose that $\widetilde{M}$ is endowed with a Riemannian metric $\widetilde{\mathbf{a}}=\left(\widetilde{a}_{i j}(x)\right)$ and a non-zero 1-form $\widetilde{\mathbf{b}}=\left(\widetilde{b}_{i}(x)\right)$ satisfying

$$
\|\widetilde{\mathbf{b}}\|^{2}=\widetilde{a}^{i j}(x) \widetilde{b}_{i}(x) \widetilde{b}_{j}(x)<1 \text {. }
$$

Then the function

$$
\widetilde{F}(x, y)=\sqrt{\widetilde{a}_{i j}(x) y^{i} y^{j}}+\widetilde{b}_{i}(x) y^{i},
$$

defines a Finsler structure on $\widetilde{M}$. According to the terminology in literature, we call $\widetilde{\mathbb{F}}^{m+p}=\left(\widetilde{M}, \widetilde{F}, \widetilde{a}_{i j}, \widetilde{b}_{i}\right)$ a proper Randers manifold. By using $\widetilde{\mathbf{a}}$ and $\widetilde{\mathbf{b}}$ we define the 1 -form

$$
\widetilde{\theta}=\widetilde{b}^{i}\left(\widetilde{b}_{i \mid j}-\widetilde{b}_{j \mid i}\right) d x^{j}
$$

where "|" denotes the covariant derivative with respect to the Levi-Civita connection on $(\widetilde{M}, \widetilde{\mathbf{a}})$.

Now, we consider the $(2 n+1)$-dimensional unit sphere $S^{2 n+1}$ and recall that Tanno [16] has proved that $S^{2 n+1}$ is a Sasakian space form of constant $\varphi$-sectional curvature $c>-3$. Then it was proved by Bejancu and Farran [6] that for any constant $K>0$ there exists a proper Randers metric of constant flag curvature $K$ and with $\widetilde{\theta}=0$ on $S^{2 n+1}$. The Finsler manifold $\widetilde{\mathbb{F}}^{2 n+1}=\left(S^{2 n+1}, c, K\right)$ is called a Randers $(c, K)$-sphere. The following classification theorem is useful in our study.

Theorem 3.7. (Bejancu-Farran [6]) Let $\widetilde{\mathbb{F}}^{m+p}=\left(\widetilde{M}, \widetilde{F}, \widetilde{a}_{i j}, \widetilde{b}_{i}\right)$ be a proper Randers manifold, where $(\widetilde{M}, \widetilde{\mathbf{a}})$ is a simply connected and complete Riemannian manifold. Suppose that $\widetilde{\mathbb{F}}^{m+p}$ is of positive constant flag curvature $K$ and that $\widetilde{\theta}=0$ on $\widetilde{M}$. Then $m+p$ must be an odd number $2 n+1$, and $\widetilde{\mathbb{F}}^{2 n+1}$ is Finsler isometric to the Randers $(c, K)$-sphere $\bar{F}^{2 n+1}=\left(S^{2 n+1}, c, K\right)$ where $c=1-4\|\mathbf{b}\|^{2}$.

Let $\mathbb{F}^{m}=(M, F)$ be a Finsler submanifold of a proper Randers manifold $\widetilde{\mathbb{F}}^{m+p}=$ $\left(\widetilde{M}, \widetilde{F}, \widetilde{a}_{i j}, \widetilde{b}_{i}\right)$. Suppose that the structure vector field $\widetilde{b}^{i}=\widetilde{a}^{i j} \widetilde{b}_{j}$ of $\widetilde{\mathbb{F}}^{m+p}$ is tangent to $M$. Then $\mathbb{F}^{m}$ inherits a Randers structure given by

$$
\text { (a) } a_{\alpha \beta}=\widetilde{a}_{i j} B_{\alpha}^{i} B_{\beta}^{j} \text { and (b) } b_{\alpha}=\widetilde{b}_{i} B_{\alpha}^{i} \text {. }
$$

Moreover, we prove the following.

Theorem 3.8. Let $\mathbb{F}^{m}=(M, F)$ be a totally geodesic Finsler submanifold of a Randers $(c, K)$-sphere $\widetilde{\mathbb{F}}^{2 n+1}=\left(S^{2 n+1}, c, K\right)$, such that $\left(M, a_{\alpha \beta}\right)$ is a simply connected and complete Riemannian manifold, and the structure vector field of $\widetilde{\mathbb{F}}^{2 n+1}$ is tangent to $M$. Then $m$ is an odd number $2 q+1$ and $\mathbb{F}^{2 q+1}$ is Finsler isometric to a Randers $(c, K)$-sphere $\overline{\mathbb{F}}^{2 q+1}=\left(S^{2 q+1}, c, K\right)$.

Proof. First, we note that $\mathbb{F}^{m}$ is a proper Randers manifold with the Riemannian metric and 1-form given by (3.32). Then, by using (3.32b) we deduce that

$$
b_{\alpha \mid \beta}=B_{\alpha}^{i} B_{\beta}^{j} \widetilde{b}_{i \mid j},
$$

where the covariant derivatives are taken with respect to the Levi-Civita connections on the Riemannian manifolds $\left(M, a_{\alpha \beta}\right)$ and $\left(S^{2 n+1}, \widetilde{a}_{i j}\right)$. By using (3.33) and taking into account that $\widetilde{\theta}=0$ on $S^{2 n+1}$, we deduce that

$$
\begin{aligned}
\theta=b^{\alpha}\left(b_{\alpha \mid \beta}-b_{\beta \mid \alpha}\right) d u^{\beta} & =b^{\alpha} B_{\alpha}^{i} B_{\beta}^{j}\left(\widetilde{b}_{i \mid j}-\widetilde{b}_{j \mid i}\right) d u^{\beta} \\
& =\widetilde{b}^{i}\left(\widetilde{b}_{i \mid j}-\widetilde{b}_{j \mid i}\right) d x^{j}=0 .
\end{aligned}
$$


Also, by Corollary 3.1 we infer that $\mathbb{F}^{m}$ is a Finsler manifold of constant flag curvature $K$. Finally, we apply Theorem 3.7 and obtain the assertion of the present theorem.

The next corollary follows immediately from Theorem 3.8.

Corollary 3.2. There exist no totally geodesic even dimensional Finsler submanifolds of a Randers $(c, K)$-sphere $\widetilde{\mathbb{F}}^{2 n+1}=\left(S^{2 n+1}, c, K\right)$ which are tangent to the structure vector field of $\widetilde{\mathbb{F}}^{2 n+1}$.

Remark 3.2. We note that the Finsler structure of a Randers $(c, K)$-sphere is never a Riemannian structure. This is because $\widetilde{\mathbf{b}}$ is nowhere zero on $S^{2 n+1}$. Thus the results in Theorem 3.8 and Corollary 3.2 cannot be applied to Riemannian geometry.

\section{REFERENCES}

[1] Akbar-Zadeh, H., Sur les sous-variétés des variétés finsleriennes, C.R. Acad. Sci. Paris, 266 (1968), 146-148.

[2] Bao, D., Chern, S.S. and Shen, Z., An Introduction to Riemann-Finsler Geometry, Graduate Text in Math., 200, Springer, Berlin, 2000.

[3] Barthel, W., Über die Minimalflächen in gefaserten Finslerräumen, Ann. di Mat., 36 (1954), 159-190.

[4] Bejancu, A., Special immersions of Finsler spaces, Stud. Cercet. Mat., 39 (1987), 463-487.

[5] Bejancu, A., Finsler Geometry and Applications, Ellis Horwood, New York, 1990.

[6] Bejancu, A. and Farran, H.R., On the classification of Randers manifolds of constant curvature, Bull. Math. Soc. Sci. Math. Roumanie, 52 (100), No. 3, 2009, 227-239.

[7] Bejancu, A. and Farran, H.R., Geometry of Pseudo-Finsler Submanifolds, Kluwer Academic Publishers, Dordrecht, 2000.

[8] Comic, I., The intrinsic curvature tensors of a subspace in a Finsler space, Tensor, N.S., 24 (1972), 19-28.

[9] Haimovici, M., Variétés totalement extrémales et variétés totalement géodésiques dans les espaces de Finsler, Ann. Sci. Univ. Jassy, 25 (1939), 559-644.

[10] Matsumoto, M., The induced and intrinsic Finsler connections of a hypersurface and Finslerian projective geometry, J. Math. Kyoto Univ., 25 (1985), 107-144.

[11] Matsumoto, M., Theory of Y-extremal and minimal hypersurfaces in a Finsler space, J. Math. Kyoto Univ., 26 (1986), 647-665.

[12] Matsumoto, M., Foundations of Finsler Geometry and Special Finsler Spaces, Kaiseisha Press, Saikawa, Ōtsu, 1986.

[13] Miron, R., A non-standard theory of hypersurfaces in Finsler spaces, An. St. Univ. "Al.I. Cuza" Iasi, 30 (1974), 35-53.

[14] Rund, H., The Differential Geometry of Finsler Spaces, Grundlehr. Math. Wiss., 101, Springer, Berlin, 1959.

[15] Shen, Z., On Finsler geometry of submanifolds, Math. Ann., 311 (1998), 549-576.

[16] Tanno, S., Sasakian manifolds with constant $\varphi$-holomorphic sectional curvature, Tôhoku Math. J., 21 (1969), 501-507.

[17] Varga, O., Über den inneren und induzierten Zusammenhang für Hyperflächen in Finslerschen Räumen, Publ. Math. Debrecen, 8 (1961), 208-217.

[18] Wegener, J.M., Hyperfächen in Finslerschen Räumen als Transversalflächen einer Schar von Extremalen, Monatsh. Math. Phys., 44 (1936), 115-130.

Department of Mathematics, and Computer Science, Kuwait University, P.O. Box 5969, SAFAT 13060, KUWAIT.

E-mail address: aurel.bejancu@ku.edu.kw, hani.farran@ku.edu.kw 